\title{
Fobias específicas: classificação baseada na fisiopatologia
}

\author{
Specific phobias: a pathophysiology based classification
}

\author{
RenATO T. RAMOS ${ }^{1}$ \\ 'Livre-docente, chefe da Unidade de Ansiedade e Depressão do IPq-HC-FMUSP e pesquisador do Laboratório de Psicofarmacologia e Psicopatologia Experimental (LIM-23). \\ Recebido: 11/05/2007 - Aceito: 29/05/2007
}

As fobias específicas são atualmente agrupadas em subtipos que incluem fobias de animais, ambientes naturais (altura, tempestades e água), sangue e ferimentos e fobias situacionais (aviões, elevadores, locais fechados etc.). O objetivo desse tipo de classificação é facilitar o diagnóstico e evitar o uso desnecessário de extensas listas de situações ou objetos. No entanto, ao se adotar subtipos de fobias, supõe-se que os medos colocados dentro de um mesmo grupo devam ser semelhantes entre si, tanto em termos de mecanismos fisiopatológicos quanto em termos de resposta a tratamentos. Esse cuidado é fundamental, pois muitos estudos acerca do tratamento das fobias específicas são baseados em um ou outro tipo de sintoma (medo de altura, por exemplo) e seus resultados são generalizados para os demais tipos de fobia (Choy et al., 2007).

Esta breve revisão visa a chamar a atenção para as evidências de que os atuais subtipos de fobias específicas talvez não sejam constituídos por quadros clínicos tão homogêneos quanto se poderia pensar. O subgrupo dos medos de ambientes naturais que incluem as fobias de altura, tempestades e água é particularmente interessante para esta discussão.

Diversos estudos pautados em análises fatoriais serviram de base para a classificação atual das fobias específicas. No entanto, os medos de altura, tempestades e água não costumam aparecer dentro do mesmo grupo ou cluster. Cox et al. (2003), por exemplo, investigaram um possível caráter hierárquico dos sintomas fóbicos a partir de dados do U.S. National Comorbidity Survey. A análise de componentes principais indicou uma solução com cinco agrupamentos que incluíram: agorafobia (locais públicos, multidões, permanecer numa fila, viajar de carro ou ônibus e afastar-se de casa); medo de falar em público; medo de ser observado (comer, escrever ou usar o banheiro público); medo de ameaças (sangue, agulhas, tempestades e trovões, animais, ficar só e medo de locais fechados) e medo de altura e água (piscinas, lagos e rios). Nota-se aqui que a agorafobia emerge como uma categoria consistente, mas o agrupamento do medo de sangue e agulhas com o medo de tempestades não parece fazer sentido clínico.

Essa falta de um agrupamento natural das fobias específicas em subtipos robustos pode estar ligada a algumas características clínicas de cada tipo de medo. Em primeiro lugar, observa-se prevalência semelhante entre homens e mulheres do medo de altura, o que não ocorre com os medos de tempestade e água, em que a prevalência em mulheres costuma ser mais que o dobro da dos homens (Curtis et al., 1990). Em segundo lugar, o medo de altura caracteriza-se por uma distribuição bimodal em relação à idade de início dos sintomas, com picos de ocorrência entre os 5 e 7 anos e por volta dos 14 anos de idade, um padrão que não é encontrado nas demais fobias (Curtis et al., 1990). Finalmente, existem evidências de que esses três tipos de fobia se desenvolvam a partir de diferentes mecanismos fisiológicos e psicológicos e as informações disponíveis acerca de sua forma de aquisição sustentam essa hipótese.

Os mecanismos de aquisição das fobias ainda são foco de discussão e existem evidências de que alguns medos sejam adquiridos por condicionamento ou outras formas de aprendizado, enquanto outros surgem de forma espontânea ou não associativa (Mineka e Ohman, 2002). A forma não associativa de aquisição de medos tem sido considerada como indicação de que certas fobias teriam uma origem inata e estariam ligadas a situações ou estímulos considerados aversivos em termos evolutivos (Poulton e Menzies, 2002).

Menzies e Clarke (1993) investigaram a origem do medo em 50 crianças com fobia de água. Em apenas um dos casos, os pais puderam lembrar-se da ocorrência 
de um evento traumático associado ao início do medo. Poulton et al. (1999) examinaram a influência da experiência de nadar no desenvolvimento do medo de água em indivíduos de 18 anos de idade que participaram do Dunedin Multidisciplinary Health and Development Study, um estudo de coorte que acompanhou 1.139 crianças do nascimento até os 18 anos de idade, com avaliações a cada dois anos. Identificou-se fobia de água em 12 de 1.008 indivíduos (1,3\%) avaliados ao final do estudo e nenhum dos seguintes eventos foi significativamente mais frequiente neste grupo em relação aos indivíduos sem sintomas fóbicos: ter caído ou mergulhado acidentalmente entre os 3 e os 5 anos de idade; ter sido exposto ocasionalmente à água entre as idades de 7 e 9 anos; ter sido socorrido em virtude de dificuldades por imersão na água entre os 7 e os 9 anos de idade.

Tem-se relatado um padrão não associativo semelhante para a aquisição do medo de altura. Estudando universitários com sintomas de fobia de altura, Menzies e Clarke (1995) documentaram a ocorrência de eventos traumatizantes nítidos como associados ao início dos sintomas em apenas 9 dos 50 (18\%) indivíduos. Ainda em relação ao medo de altura, Poulton et al. (1998) não verificaram relação significativa entre a ocorrência de quedas graves na infância e a ocorrência de fobia de altura em idades de 11 a 18 anos. Este estudo usou a mesma amostra do Estudo de Dunedin e dos 55 indivíduos (avaliados aos 18 anos de idade) que sofreram quedas com ferimentos graves ao longo da vida e nenhum caso desenvolveu sintomas fóbicos de altura. $\mathrm{Na}$ verdade, a ocorrência de quedas não triviais parece ser maior em indivíduos que não apresentam fobia de altura (Poulton et al., 2001), sugerindo que a esquiva de locais altos surge precocemente e de certa forma previne a ocorrência de quedas.

Em contraste com a falta de eventos traumáticos ligados à aquisição das fobias de água e altura, eventos traumatizantes parecem ter um papel relevante na aquisição da fobia de tempestades. Westefeld (1996) estudou 81 indivíduos (46 mulheres) com sintomas graves de medo de tempestades. Desses indivíduos, 65 (80\%) relataram que os sintomas fóbicos começaram após a exposição a algum tipo de tempestade intensa e 12 desses sujeitos relataram que tal experiência ocorreu entre as idades de 5 e 15 anos. Apenas 4 sujeitos relataram não ter idéia de como seus sintomas fóbicos haviam começado.

Além desses dados essencialmente epidemiológicos, existem evidências de que anormalidades fisiológicas contribuam para o caráter heterogêneo do subtipo ambientes naturais das fobias específicas. Especificamente, a fobia de altura, assim como a agorafobia, parece relacionar-se a anormalidades do controle de postura e equilíbrio, característica esta não encontrada nos demais medos deste grupo. Resumidamente, indivíduos com medo de altura teriam algum tipo de deficiência no controle vestibular da postura que os tornaria mais dependentes de informações visuais ou proprioceptivas para manter o equilíbrio. Em lugares altos, há poucas referências visuais, o que levaria à instabilidade postural e ao surgimento de sintomas vegetativos secundários a uma situação de perigo real de queda (Jacob et al., 1993; Ramos et al., 1997; Jacob et al., 2001; Ramos, 2006). Essa hipótese poderia explicar o surgimento do medo de altura independentemente da ocorrência de eventos traumáticos. Na verdade, indivíduos com esse tipo de problema tenderiam a evitar lugares altos desde fases mais precoces do desenvolvimento do controle postural. Existem evidências de que por volta dos 7 anos de idade, ocorra um período natural de dependência visual mais intensa para a manutenção do equilíbrio, dependência esta que desaparece por volta dos 14 a 15 anos de idade (Assaiante e Amblard, 1995). Anormalidades nesse padrão de desenvolvimento poderiam levar ao desencadeamento de medo exagerado de altura por mecanismos primariamente biológicos mais do que psicológicos.

Este tipo de discussão é importante para o entendimento da fisiopatologia das fobias e, mais ainda, para o aperfeiçoamento de suas formas de tratamento. A terapia de exposição in vivo, por exemplo, é muito eficaz no tratamento das fobias específicas, mas faltam estudos que investiguem as razões para as altas taxas de recusa e abandono do tratamento. Provavelmente medos ligados a eventos traumáticos levem o paciente a deixar o tratamento por razões diferentes daqueles medos com que o indivíduo nunca chegou a experimentar um evento realmente ameaçador. Além disso, a terapia cognitiva não parece trazer benefícios adicionais sobre a terapia de exposição no tratamento das fobias de ambientes naturais, mas parece ser vantajosa quando aplicada a pessoas com claustrofobia e fobia dental, condições muitas vezes ligadas a eventos traumáticos (Choy et al., 2007).

Por fim, a presença de anormalidades do controle postural leva à hipótese de que a terapia de reabilitação vestibular possa ser particularmente útil no tratamento da fobia de altura (Whitney et al., 2005). Muitos dos exercícios propostos para expor o paciente a sensações corporais desagradáveis (na exposição a estímulos internociceptivos, por exemplo) são muito semelhantes àqueles usados para melhorar o funcionamento vestibular. Avanços no conhecimento desses mecanismos talvez levem a mudanças nas futuras classificações e nas formas de tratamento das fobias específicas.

\section{Referências}

Assaiante, C.; Amblard, B. - An ontogenetic model for the sensorimotor organization of balance control human. Human Movement Science 14: 13-43, 1995.

Choy, Y.; Fyer, A.J.; Lipsitz, J.D. - Treatment of specific phobia in adults. Clin Psychol Rev 27(3): 266-286, 2007.

Cox, B.J.; McWilliams, L.A.; Clara, I.P.; Stein, M.B. - The structure of feared situations in a nationally representative sample. J Anxiety Disord 17(1): 89-101, 2003.

Curtis, G.C.; Hill, E.M.; Lewis, J.A. - Heterogeneity of DSM-III simple phobia and the simple phobia/agoraphobia boundary: evidence from the ECA 
Study (preliminary report to the Simple Phobia Subcommittee of the DSM-IV Anxiety Disorders Work Group. DSM-IV sourcebook. In: Widiger, T.A., Frances, A.J.; Pincus, H.A. et al. - Washington, DC, American Psychiatric Association. 2: 480-482, 1990

Jacob, R.G.; Whitney, S.L.; Detweiler-Shostak, G.; Furman, J.M. - Vestibular rehabilitation for patients with agoraphobia and vestibular dysfunction: a pilot study. J Anxiety Disord 15(1-2): 131-146, 2001.

Jacob, R.G.; Woody, S.R.; Clark, D.; Lilienfeld, S.; Hirsch, B.E.; Kucera, G.; Furman, J.M.; Durrant, J.D. - Discomfort with space and motion: a possible marker of vestibular dysfunction assessed by the situational characteristics questionnaire. Journal of Psychopathology and Behavior Assessment 15: 299-324, 1993.

Menzies, R.G.; Clarke, J.C. - A comparison of in vivo and vicarious exposure in the treatment of childhood water phobia. Behav Res Ther 31(1): 9-15, 1993.

Menzies, R.G.; Clarke, J.C. - The etiology of acrophobia and its relationship to severity and individual response patterns. Behavior Research and Therapy 33(7): 795-803, 1995.

Mineka, S.; Ohman, A. - Born to fear: non-associative vs associative factors in the etiology of phobias. Behav Res Ther 40(2): 173-184, 2002.

Poulton, R.; Menzies, R.G. - Fears born and bred: toward a more inclusive theory of fear acquisition. Behav Res Ther 40(2): 197-208, 2002.
Poulton, R.; Davies, S.; Menzies, R.G.; Langley, J.D.; Silva, P.A. - Evidence for a non-associative model of the acquisition of a fear of heights. Behavior Research and Therapy 36(5): 537-544, 1998.

Poulton, R.; Menzies, R.G.; Craske, M.G.; Langley, J.D.; Silva, P.A. - Water trauma and swimming experiences up to age 9 and fear of water at age 18: a longitudinal study. Behav Res Ther 37(1): 39-48, 1999.

Poulton, R.; Waldie, K.E.; Menzies, R.G.; Craske, M.G.; Silva, P.A. - Failure to overcome "innate" fear: a developmental test of the non- associative model of fear acquisition. Behavior Research and Therapy 39(1): 29-43, 2001.

Ramos, R.T. - Antidepressants and dizziness. J Psychopharmacol 20(5): 708-713, 2006.

Ramos, R.T.; Jacob, R.G.; Lililenfeld, S.O. - Space and motion discomfort in Brazilian versus American patients with panic disorder. J Anxiety Disord 11: 131-139, 1997.

Westefeld, J.S. - Severe weather phobia: an exploratory study. J Clin Psychol 52(5): 509-515, 1996.

Whitney, S.L.; Jacob, R.G.; Sparto, P.J.; Olshansky, E.F.; Detweiler-Shostak G.; Brown, E.L.; Furman, J.M. - Acrophobia and pathological height vertigo: indications for vestibular physical therapy?. Physical Therapy 85: 443-458, 2005. 